\title{
Two-way transparency in the light-matter interaction: Optical precursors with electromagnetically induced transparency
}

\author{
Heejeong Jeong ${ }^{1, *}$ and Shengwang $\mathrm{Du}^{2, \dagger}$ \\ ${ }^{1}$ Thayer School of Engineering, Dartmouth College, Hanover, New Hampshire 03755, USA \\ ${ }^{2}$ Department of Physics, The Hong Kong University of Science and Technology, Clear Water Bay, Kowloon, Hong Kong, China
}

(Received 31 August 2008; published 8 January 2009)

\begin{abstract}
We theoretically study classical step-modulated pulse propagation in an optically thick medium with electromagnetically induced transparency (EIT) using a fast Fourier transform and a proposed hybrid-asymptotic analysis. The $100 \%$ transmitted leading edge traveling at $c$ consists of Sommerfeld-Brillouin precursors. The main signal, falling inside of the narrow EIT window, experiences a steep dispersion and slow group velocity. We suggest active control of the precursors and main signal separately by adjusting the parameters of the medium and incident pulse.
\end{abstract}

DOI: 10.1103/PhysRevA.79.011802

PACS number(s): 42.50.Gy, 42.50.Nn, 42.25.Bs

The effort to achieve active control of the light-matter interaction has brought on the recent developments of nanophotonics for fast and advanced communication and information technology [1]. Electromagnetically induced transparency (EIT) [2], for example, switches light transmission on and off with an auxiliary control beam. For a three-level system as shown in Fig. 1(a), a coupling laser field $\omega_{c}$ rends the medium transparent at the otherwise probe absorptionresonance frequency $\omega_{0}$. The steep dispersion of the linear susceptibility within a narrow transparent window leads to slow and ultraslow light $[3,4]$. Note that if the coupling laser is turned off, it reduces to a two-level system (classically single-Lorentz dielectric), as in the blue dashed line in Figs. 1(b) and 1(c).

If EIT is considered as "steady-state transparency," there is "transient transparency" in the light-matter interaction, optical precursors, introduced about a century ago by Sommerfeld and Brillouin to prove no light component travels faster than $c$ [5]. When a monochromatic wave is abruptly switched on and shined on a single-Lorentz dielectrics, the front edge of a step-modulated pulse ideally does not interact with the medium and travels at $c$ because of the finite response time. The front edge is followed by high- and lowfrequency component consisting of transients: Sommerfeld and Brillouin precursors, respectively. Contrary to predicted difficulties in detection, a direct observation of optical precursors was recently made by using cold atoms [6]. The novel characteristics of large-peak and significantly longlasting transients (precursors) have also been verified theoretically [7]. Optical precursors are currently of increasing interest as they are connected to biomedical imaging and optical communication applications because they can penetrate deeper into a material $[8,9]$.

However, optical precursors in classical pulse propagation have been always focused on single or double Lorentz dielectric media and have not been studied in an EIT system. Most recently optical precursors at the biphoton level have been observed in an EIT system [10,11]. The ballistic char-

\footnotetext{
*jhj413@gmail.com

†dusw@ust.hk
}

acteristic of optical precursors is measured as a sharp peak at the leading edge of the biphoton correlated wave packet generated using EIT and slow light. The motivation of this work is to study how optical precursors form in classical pulse propagation through an EIT medium and examine whether they differ from the biphoton case.

In this Rapid Communication, we discuss classical stepmodulated pulse propagation through an EIT medium based on the parameters in Refs. [10,11]. We implement two numerical methods: fast Fourier transform (FFT) and hybridasymptotic analysis. The FFT is used to evaluate total transmission, and the hybrid-asymptotic method is proposed to identify the main signal and Sommerfeld-Brillouin precursors. The result shows interesting properties of the main signal's propagation in slow-or fast-light media. The steadystate transmission and the group delay of the main signal are affected by medium parameters, such as the optical depth $\alpha_{0} z$ and coupling Rabi frequency $\Omega_{c}$. This suggests a clear interpretation of the main signal's arrival, contrary to the compli-

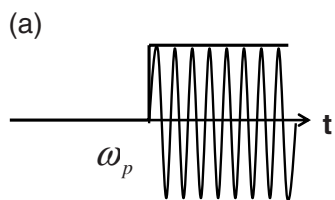

Incident pulse

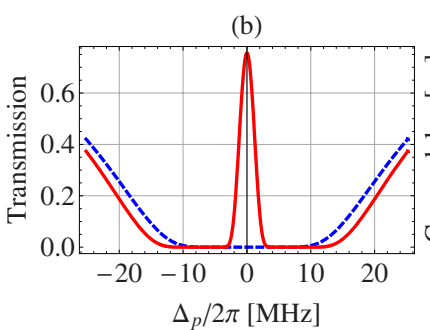

FIG. 1. (Color online) Optical pulse propagation through an EIT medium. (a) Illustration of a step-modulated incident pulse and EIT level scheme. (b) Transmission and (c) group delay for EIT $\left(\Omega_{c}\right.$ $=4.2 \delta$, red solid line) and for the single-Lorentz medium case $\left(\Omega_{c}\right.$ $=0$, blue dashed line). $\omega_{p}$ is the incident pulse carrier frequency, and $\Delta_{p}=\omega_{p}-\omega_{0}$ is the pulse carrier detuning. 

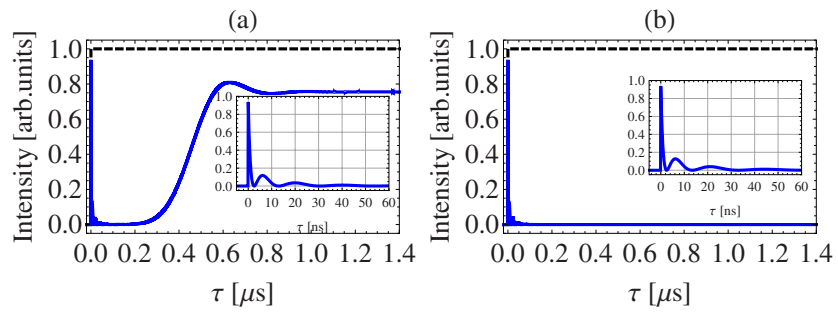

FIG. 2. (Color online) Total step pulse transmission (solid line) and Sommerfeld-Brillouin precursor (inset) in (a) an EIT medium $\left(\Omega_{c}=4.20 \delta\right)$ and (b) for the EIT-off case $\left(\Omega_{c}=0\right)$. The dashed line denotes the input step-modulated pulse. Here, we take $\delta=2 \pi$ $\times 3 \mathrm{MHz}, \delta_{12}=0.02 \delta, \alpha_{0} z=62$, and $\Delta_{p}=0$.

cated expression for the far off-resonance case in conventional asymptotic saddle-point analysis on optical precursors $[5,12]$.

The general description of transmitted electromagnetic field is usually obtained by Fourier analysis accompanyed by spectral medium characteristics: complex index of refraction, $n(\omega)=\sqrt{1+\chi(\omega)}$, where $\chi(\omega)$ is the linear susceptibility. For an EIT medium, the probe atomic transition exhibits a rapidly varying refractive index whose dispersive properties are mediated by the coupling field $[2,4,13-15]$. The linear susceptibility is given by [16]

$$
\chi(\omega)=\frac{c \alpha_{0}}{\omega_{0}} \frac{4\left(\Delta+i \delta_{12}\right) \delta}{\Omega_{c}^{2}-4\left(\Delta+i \delta_{12}\right)(\Delta+i \delta)},
$$

where $\Delta \equiv \omega-\omega_{0}$ is the frequency detuning, $\alpha_{0} z=62$ is the optical depth, $\delta=2 \pi \times 3 \mathrm{MHz}$ is half of the natural linewidth (the resultant coherence decay time of the excited level $|3\rangle$ is $\left.T_{2}=1 / \delta=53 \mathrm{~ns}\right), \delta_{12}=0.02 \delta$ is the dephasing rate between the two ground levels, and $\Omega_{c}=4.20 \delta$ is the coupling laser Rabi frequency. The transmitted field is given as an integral form

$$
E(z, t)=\frac{1}{2 \pi} \int_{-\infty+i \epsilon}^{\infty+i \epsilon} E(0, \omega) e^{(z / c) \phi(\omega, t)} d \omega,
$$

where $E(0, \omega)$ is the spectrum of the initial step-modulated pulse, $E(0, t)=E_{0} \Theta(t) e^{-i \omega_{p} t}$, and the complex phase of the integrand is $\phi(\omega, t)=i c[k(\omega) z-\omega t] / z$.

We first study the on-resonance case-i.e., $\Delta_{p}=0$. The total transmission of the step-modulated input pulse through an optically thick medium $\left(\alpha_{0} z=62\right)$ is evaluated numerically based on Eq. (2) using FFT, as shown in Fig. 2. The solid lines indicate total transmission, and the insets show earlier temporal evolution as a function of retarded time $\tau$ $\equiv t-z / c$, which is the Sommerfeld-Brillouin precursor discussed later. With $\Omega_{c}=4.20 \delta$ [Fig. 2(a)], the steady-state transmission arrives with a group delay of 370 ns. Approximately $80 \%$ of the transmission agrees with the EIT transmission in Fig. 1(b). When the coupling beam is turned off $\left(\Omega_{c}=0\right)$, the steady-state part is totally absorbed by the optically thick medium. The transient part arriving at $c$, however, is not affected by medium properties, thereby being interpreted as optical precursors.
Optical precursors emerging from the optically thick single-Lorentz medium are well defined and analyzed by the steepest-decent method associated with asymptotic analysis $[5,12]$. Equation (2) is asymptotically $\left(z \gg \alpha_{0}^{-1}\right)$ evaluated by the deformation on the contour of the integral near the saddle points $\omega_{s p}$ obtained from $\left.\partial_{\omega} \phi(\omega, t)\right|_{\omega_{s p}}=0$. Since the quadratic expansion fails within the narrow EIT transparency window where $\left.\partial_{\omega}^{2} \phi(\omega, t)\right|_{\omega_{0}}=0$, we separate two regions as suggested in Ref. [11]. Here, we propose a hybrid-asymptotic analysis, in which we use two different methods for two different regimes: EIT transparency window, $\left[\omega_{0}-\Delta_{e}, \omega_{0}\right.$ $\left.+\Delta_{e}\right]$, its complement set. The quantity $\Delta_{e} \sim \Omega_{c}$ is determined by the EIT transmission profile where the frequencies of $\omega_{0}-\Delta_{e}$ and $\omega_{0}+\Delta_{e}$ fall into the opacity window. For the complement set, where the asymptotic analysis is well defined and used to identify Sommerfeld-Brillouin precursors, we use a quadratic approximation near saddle points of the phase [17]. A new concept here compared to the previous method [11] is to exploit impulse response method (Green's function) instead of numerical FFT [11] to obtain the main signal.

To obtain Sommerfeld-Brillouin precursors, we evaluate saddle points satisfying $\left.\partial_{\omega} \phi(\omega, t)\right|_{\omega_{s p}}=0$ and obtain

$$
\frac{1}{\left.\partial_{\omega} k(\omega)\right|_{\omega_{s p}}}=\frac{z}{t} \text {. }
$$

At high optical depth $\left(\alpha_{0} z\right)$, only the saddle points falling outside of the opacity window contribute to the precursor fields. When $\Delta_{s p}=\omega_{s p}-\omega_{0} \gg \Omega_{c}$, a single-resonance non-EIT process dominates and we can approximately treat the medium as a two-level system. Then approximated solutions are obtained as

$$
\Delta_{s p}^{ \pm} \equiv \omega_{s p}^{ \pm}-\omega_{0} \simeq \pm \xi(t)-i \delta,
$$

where $\xi(t) \equiv \sqrt{\frac{\alpha_{0} z \delta}{2(t-z / c)}}$. Under the quadratic approximation, we obtain asymptotic expressions of Sommerfeld $E_{S}(z, t)$ and Brillouin $E_{B}(z, t)$ precursors as

$$
\begin{aligned}
& E_{S}(z, t)=\frac{i E_{0}}{\sqrt{2 \pi} \Delta_{s p}^{+}} \frac{e^{(z / c) \phi\left(\omega_{s p}^{+}, t\right)-i \pi / 4}}{\sqrt{\alpha_{0} z \delta / \xi(t)^{3}}}, \\
& E_{B}(z, t)=\frac{i E_{0}}{\sqrt{2 \pi} \Delta_{s p}^{-}} \frac{e^{(z / c) \phi\left(\omega_{s p}^{-}, t\right)+i \pi / 4}}{\sqrt{\alpha_{0} z \delta / \xi(t)^{3}}} .
\end{aligned}
$$

Now we turn our attention to the main signal $E_{C}(z, t)$. Here we define the Green's function (impulse response) of the EIT window $\left[\omega_{0}-\Delta_{e}, \omega_{0}+\Delta_{e}\right]$ as

$$
G_{E I T}(z, t)=\frac{1}{2 \pi} \int_{\omega_{0}-\Delta_{e}}^{\omega_{0}+\Delta_{e}} e^{i[k(\omega) z-\omega t]} d \omega .
$$

For the case shown in Fig. 1(b), we take $\Delta_{e}=\Omega_{c} / 2=2 \pi$ $\times 6.4 \mathrm{MHz}$. The main signal $E_{C}(z, t)$ can be expressed as a convolution of Green function, Eq. (7), and the input stepmodulated pulse $E(0, t)$ :

$$
E_{C}(z, t)=\int_{-\infty}^{\infty} G_{E I T}(z, t-\tau) E(0, \tau) d \tau
$$




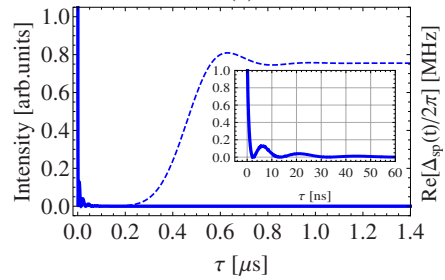

FIG. 3. (Color online) Hybrid (asymptotic-convolution) method [Eqs. (5)-(9)]. (a) Evaluation of Sommerfeld-Brillouin precursors $\left[E_{S}(z, t)+E_{B}(z, t)\right.$, solid line $]$ and main signal $\left[E_{C}(z, t)\right.$, dashed line $]$ in the EIT medium. (b) Frequencies of Sommerfeld (blue solid line) and Brillouin (red dashed line) precursors as functions of retarded time $\tau=t-z / c$ for the on-resonance case $\left(\Delta_{p}=0\right)$. The parameters are $\delta=2 \pi \times 3 \mathrm{MHz}, \delta_{12}=0.02 \delta, \Omega_{c}=4.20 \delta$, and $\alpha_{0} z=62$.

$$
=\int_{0}^{\infty} G_{E I T}(z, t-\tau) d \tau .
$$

Therefore, the total transmitted field is obtained from Eqs. (5)-(9): $E(z, t)=E_{S}(z, t)+E_{B}(z, t)+E_{C}(z, t)$. The corresponding normalized intensity is shown in Fig. 3(a). The solid line denotes the intensity of total precursors, $\left|E_{S}(z, t)+E_{B}(z, t)\right|^{2}$, and the dotted line represents the main signal intensity, $\left|E_{C}(z, t)\right|^{2}$. Because of the distinct separation between the transient and steady-state parts, there is little interference between two parts. Figure 3(b) shows the frequencies of Sommerfeld (blue solid line) and Brillouin (red dashed line) precursors. The modulation of the total precursors is due to the beating between two sidebands of the pulse spectrum propagating through optically thick medium.

To investigate the role of the EIT effect on the precursors and main signal for the on-resonance case $\left(\Delta_{p}=0\right)$, we vary the optical depth $\alpha_{0} z$ and coupling Rabi frequency $\Omega_{c}$, as shown in Fig. 4. Table I shows steady-state transmissions and the group delays for four different cases: (a1) $\alpha_{0} z=30$ and $\Omega_{c}=2.35 \delta$, (a2) $\alpha_{0} z=30$ and $\Omega_{c}=4.20 \delta$, (b1) $\alpha_{0} z=53$ and $\Omega_{c}=2.35 \delta$, and (b2) $\alpha_{0} z=53$ and $\Omega_{c}=4.20 \delta$.

Sommefeld-Brillouin precursors are independent with $\Omega_{c}$ (insets of Fig. 4), but are more oscillatory as $\alpha_{0} z$ increases, which is predicted as coherent transients [18]. The main signal, however, is significantly affected by medium properties. As in Fig. 4 and Table I, the arrival of main signal is delayed for large $\alpha_{0} z$ and small $\Omega_{c}$ because of the slow-light effect. The steady-state transmission increases as $\alpha_{0} z$ decreases and

(a)

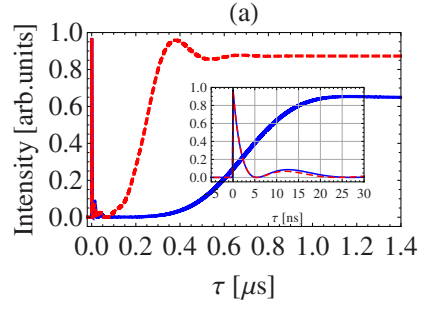

FIG. 4. (Color online) Transmission of step-modulated pulse through various EIT medium for $\Delta_{p}=0$, and different optical depths: (a) $\alpha_{0} z=30$, and (b) $\alpha_{0} z=53$. Blue solid line (red dashed line) denotes $\Omega_{c}=2.35 \delta\left(\Omega_{c}=4.20 \delta\right)$.
TABLE I. Group delays and steady-state transmission for four different cases: (a1) $\alpha_{0} z=30$ and $\Omega_{c}=2.35 \delta$, (a2) $\alpha_{0} z=30$ and $\Omega$ $=4.20 \delta$, (b1) $\alpha_{0} z=53$ and $\Omega_{c}=2.35 \delta$, and (b2) $\alpha_{0} z=53$ and $\Omega_{c}$ $=4.20 \delta$. For all cases, $\delta=2 \pi \times 3 \mathrm{MHz}$ and $\delta_{12}=0.02 \delta$ are fixed parameters.

\begin{tabular}{lcccc}
\hline \hline & $(\mathrm{a} 1)$ & $(\mathrm{a} 2)$ & (b1) & (b2) \\
\hline Group delay [ns] & 560 & 179 & 989 & 316 \\
Transmission & 0.65 & 0.87 & 0.47 & 0.79 \\
\hline \hline
\end{tabular}

as $\Omega_{c}$ increases. These behaviors are denoted as the black solid line $\left(\Delta_{p}=0\right)$ in Fig. 5 .

Figure 5 shows further analysis of group delay and steady-state transmission at the EIT window for four different pulse carrier detuning cases: $\Delta_{p} / 2 \pi=0,3.2,6.4$, and $9.5 \mathrm{MHz}$. As the optical depth $\alpha_{0} z$ increases, the steady-state transmission rapidly decreases [Fig. 5(a)], and group delay increases [Fig. 5(c)] except for $\Delta_{p} / 2 \pi=6.4 \mathrm{MHz}$. Note that the boundary of the EIT is located at $\Delta_{e} / 2 \pi=\Omega_{c} / 4 \pi$ $=6.4 \mathrm{MHz}$, where the negative group delay in the highly absorptive region results in pulse advancement. As $\Omega_{c}$ increases, the EIT window opens wide enough to transmit light even for off-resonance cases [Fig. 5(b)]. The corresponding group delay is shown in Fig. 5(d).

We compare temporal evolution of the step-modulated pulse for the case of positive group delay [Fig. 6(a), $\Delta_{p} / 2 \pi=3.2 \mathrm{MHz}$ ] and negative group delay [Fig. 6(b), $\left.\Delta_{p} / 2 \pi=6.4 \mathrm{MHz}\right]$. No pulse advancement is observed, thereby confirming that the main signal's arrival never exceeds $c$. Another notable thing is, contrary to the $\Delta_{p}=0$ case, we observe interference between the Sommerfeld-Brillouin precursors and main signal.

We note the differences between the classical pulse propagation (Fig. 4) and biphoton generation in the EIT medium [11]. In the biphoton case, the main waveform always

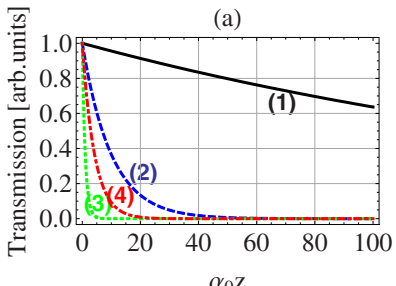

(c)

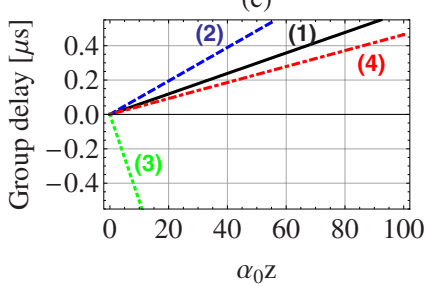

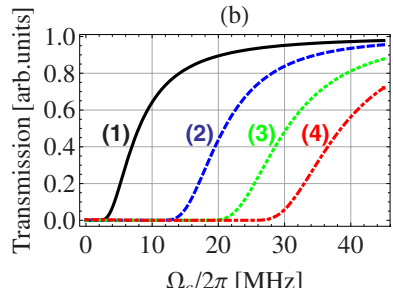

(d)

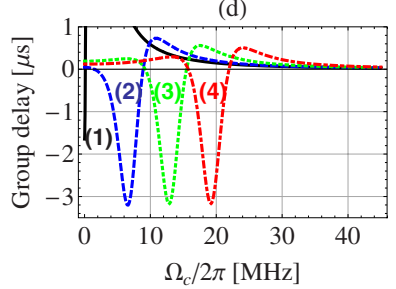

FIG. 5. (Color online) Group delay and steady-state transmission as a function of absorption depth $\alpha_{0} z$ or $\Omega_{c}$ for various pulse carrier detuning $\Delta_{p} / 2 \pi=0 \mathrm{MHz}$ (1: black solid line), $3.2 \mathrm{MHz}$ (2: blue dashed line), $6.4 \mathrm{MHz}$ (3: green dotted line), and 9.5 MHz (4: red dash-dotted line). The default parameters are $\delta=2 \pi \times 3 \mathrm{MHz}$, $\delta_{12}=0.02 \delta, \Omega_{c}=4.20 \delta$, and $\alpha_{0} z=62$. 

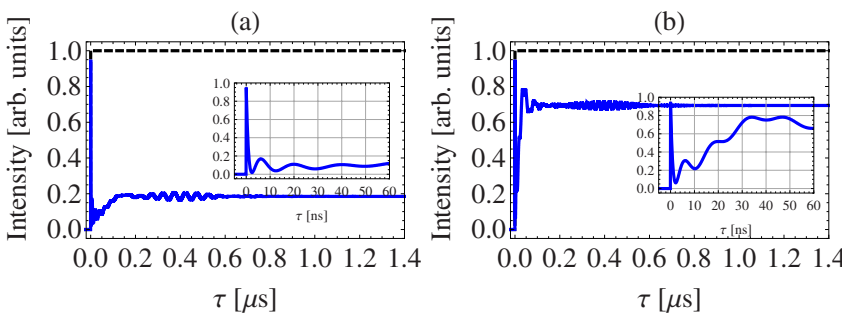

FIG. 6. (Color online) Temporal evolution of the step-modulated pulse for various detunings (a) $\Delta_{p} / 2 \pi=3.2 \mathrm{MHz}$ and (b) $6.4 \mathrm{MHz}$. Here, we use the FFT method with parameters $\delta=2 \pi \times 3 \mathrm{MHz}$, $\delta_{12}=0.02 \delta, \Omega_{c}=4.20 \delta$, and $\alpha_{0} z=62$.

switches on at the zero relative delay time and therefore it cannot be separated from the precursor field. However, in classical pulse propagation, depending on the parameters $\left(\alpha_{0} z, \Omega_{c}\right.$, and $\left.\Delta_{p}\right)$, we have active control of the interference between the main and precursor fields: in the slow-light case, the front of the main signal is always delayed and often separated from the precursors; in the fast-light case, the main signal always interferes with the precursors.

In summary, we propose a hybrid-asymptotic method to identify the main signal and Sommerfeld-Brillouin precursors when a step-modulated pulse transmits through an EIT medium. To obtain the main signal, we take the convolution of the incident pulse and the EIT window response, where the conventional asymptotic method fails. Our method agrees well with the numerical FFT results. The method provides a clear physical interpretation of the main signal's arrival compared to the complicated mathematical expressions associated with asymptotic analysis. The main signal arrives at the group velocity until it reaches the negative group index. For the negative group index $\left(\Delta_{p} / 2 \pi=6.4 \mathrm{MHz}\right)$, there is no pulse wave-front advancement as seen in Fig. 6(b), which agrees with recent experimental results [6] for an absorptive region (fast-light case) in a single-Lorentz media. Therefore, the EIT transparency window is crucial for understanding the role of group velocity on the arrival of the main signal. Our analysis proposes robust controls of transmissions or delays between the Sommerfeld-Brillouin precursors and main signal by tuning the operating parameters, which may find applications in biomedical imaging and optical communication. Meanwhile, these optical coherent transients provide spectroscopic studies in a time domain that is complementary to those made in the frequency domain.

S.D. acknowledges the start-up support from the Department of Physics at The Hong Kong University of Science and Technology.
[1] R. W. Boyd and D. J. Gauthier, Nature (London) 441, 701 (2006).

[2] S. E. Harris, Phys. Today 50(7), 36 (1997).

[3] A. Kasapi, M. Jain, G. Y. Yin, and S. E. Harris, Phys. Rev. Lett. 74, 2447 (1995).

[4] L. V. Hau, S. E. Harris, Z. Dutton, and C. H. Behroozi, Nature (London) 397, 594 (1999)

[5] L. Brillouin, Wave Propagation and Group Velocity (Academic Press, New York, 1960).

[6] H. Jeong, A. M. C. Dawes, and D. J. Gauthier, Phys. Rev. Lett. 96, 143901 (2006).

[7] W. R. LeFew, S. Venakides, and D. J. Gauthier, e-print arXiv:0705.4238.

[8] R. Albanese, J. Penn, and R. Medina, J. Opt. Soc. Am. A 6, 1441 (1989).

[9] S. H. Choi and U. Österberg, Phys. Rev. Lett. 92, 193903 (2004).
[10] S. Du, P. Kolchin, C. Belthangady, G. Y. Yin, and S. E. Harris, Phys. Rev. Lett. 100, 183603 (2008).

[11] S. Du, C. Belthangady, P. Kolchin, G. Yin, and S. Harris, Opt. Lett. 33, 2149 (2008).

[12] K. Oughstun and G. Sherman, Electromagnetic Pulse Propagation in Causal Dielectrics (Springer-Verlag, Berlin, 1994).

[13] S. E. Harris and L. V. Hau, Phys. Rev. Lett. 82, 4611 (1999).

[14] S. E. Harris, J. E. Field, and A. Kasapi, Phys. Rev. A 46, R29 (1992).

[15] M. Xiao, Y. Q. Li, S. Z. Jin, and J. Gea-Banacloche, Phys. Rev. Lett. 74, 666 (1995).

[16] D. A. Braje, V. Balić, S. Goda, G. Y. Yin, and S. E. Harris, Phys. Rev. Lett. 93, 183601 (2004).

[17] J. D. Jackson, Classical Electrodynamics, 3rd ed. (Wiley, New York, 1999).

[18] H. Jeong and U. L. Osterberg, J. Opt. Soc. Am. B 25, B1 (2008). 